CERN-TH.6552/92

CPT-92/P.E. 2715

\title{
NON-COMMUTATIVE GEOMETRY: A PHYSICIST'S BRIEF SURVEY
}

\author{
R. Coquereaux*) \\ Theoretical Physics Division, CERN \\ CH - 1211 Geneva 23
}

\begin{abstract}
This is an introduction to the old and new concepts of non-commutative (N.C.) geometry. We review the ideas underlying N.C. measure and topology, N.C. differential calculus, N.C. connections on N.C. vector bundles, and N.C. Riemannian geometry by following A. Connes' point of view.
\end{abstract}

Lectures given at the XXIIX Karpacz Winter School of Theoretical Physics

"Fields and Geometry"

Karpacz, February 1992.

*) On leave of absence from: Centre de Physique Théorique, CNRS Luminy, Case 907, F 13288

- Marseille Cedex 9 (France).

CERN-TH.6552/92

CPT-92/P.E. 2715

June 1992 


\section{Non commutative geometry: a physicist's brief survey}

\section{Foreword}

The following set of lectures is a introduction to the field of non-commutative geometry. It is supposed to be elementary, in the sense that the lectures should be easy to read, but it should lead us nevertheless to some advanced topics. This implies that we shall have to skip many important details in order to proceed. It also means that it is not possible to put everything in such a small volume. The organizers of this School hoped that a lecturer could try to make a paedagogical overview of the field, and could, at the same time, try to present some of the recent ideas of A. Connes. This is not an easy task and may be doomed to failure, but it is nevertheless the purpose of this short set of notes. Therefore I apologize for not being able to discuss here all of the many interesting topics belonging to the realm of non-commutative geometry (for instance quantum groups), first by lack of space and also because other lecturers will do it. Notice that an introductory book to non-commutative geometry already exists [1]. This book is available in French but an English version of it should appear soon [2]. The following is by no means a "summary" of this book, but a set of some paedagogical -hopefully- digressions about selected ideas to be found there. Besides, there are also a few topics not discussed in [1] but in earlier papers such as [3], topics that will also be discussed briefly. It was recently observed that non-commutative geometry offers a very nice conceptual framework for the mathematical implementation of what is usually known as the "Standard Model" of particle physics. Obtaining the classical Lagrangian of electroweak interactions from a very general mathematical construction is certainly very nice, since it gives us a new way of thinking about space and matter; but it is not clear that this is going to be the most important contribution of non-commutative geometry to physics. In any case, a very detailed account of the construction introduced in [4],[2],[3] is already available [5]. For these reasons one should not expect to find a detailed discussion of these topics here, and only a single section the last- will be devoted to them. The following is therefore only what the title suggests, namely a brief -and partial- introductory survey of non-commutative geometry, which is doomed to become obsolete soon. It should be perceived as an invitation to further reading and study. Several theoretical physicists and mathematicians share the belief that non-commutative geometry is going to change our philosophical point of view on quantum physics, and physics in general, and that it should also provide the mathematical framework in which long-standing problems such as the construction of four-dimensional non perturbative quantum field theories or even quantization of gravity, should take place. This certainly justifies the interest of physicists in learning the present subject, but this is only a hope at the moment.

\section{What is non-commutative geometry?}

In order to answer this question, we should first remember the following result (Gelfand): The definition of a given topological space $X$ (a set of "points" $x$ ) is equivalent to the definition of a commutative algebra $\mathcal{A}$. The relation between these two points of view is the following: $\mathcal{A}=C(X)$ is the algebra of complex valued functions on $X$. Conversely, $X$ is the spectrum of the algebra $\mathcal{A}$, i.e. points $x$ can be considered as irreducible representations, thanks to the magic (and obvious) relation $x[f]=f(x)$ when $f \in \mathcal{A}$. Our 
classical point of view on the Universe - even after the Einsteinian revolution- refers to "spaces", i.e. spaces of "physical points" or spaces of "events". The previous theorem says that claiming that the Universe is described by a commutative algebra is conceptually the same thing as claiming that the Universe (space or space-time) is described by a topological space (a set of points). In the case of a space (a set) $X$, it is traditional to study, in turn, measure theory, topology, differential geometry, Riemannian geometry and possibly more particular structures like a group structure or a homogeneous-space structure, etc Because of the fundamental duality between $X$ and $\mathcal{A}=C(X)$, it is clear that everything that can be written in terms of $X$ can be written in terms of $\mathcal{A}$. This will be the first task. Then one has to do so that all fundamental mathematical concepts are expressed in a way that does not require commutativity of the algebra $\mathcal{A}$. When this is possible, we just have to erase the qualifier "commutative" and promote the corresponding concepts to the realm of non-commutative algebras. One answer to the question asked previously is therefore the following: the usual geometry is the study of commutative algebras and non-commutative geometry is the study of non-commutative algebras. But this answer is not very satisfactory because what is important here is the intuitive point of view, the fact that usual geometry was taken as a guideline and that we want to think about the different features of these new mathematics as those properties of non-commutative algebras that generalize the well-known properties of usual spaces. Maybe a title such as "non-commutative mathematics" should be more appropriate, but it would cloud the fact that, in this new game, we always want to follow usual geometry as a guide.

\section{Who does non-commutative geometry?}

First, of course, we have the mathematicians themselves. One can distinguish two main lines of research ( this is often done simultaneously). First people have to develop new tools and concepts. Next, one can apply these new tools to study examples, for instance "bad" spaces (bad quotients, infinite dimensional situations) or specific non-commutative algebras.

Then we have the physicists. Historically, non-commutative geometry is not new since it is as old as quantum mechanics itself! Actually the tools created by physicists - or at least used for quite a while- have invaded the field of operator algebras. Let us just mention a few concepts: Pure states and mixtures in statistical mechanics (states on stellar algebras), equilibrium states (KMS states, Tomita-Takesaki theory and von Neumann factors), quantification of classical systems (deformation theory), renormalization of logarithmically divergent operators (Dixmier trace), Higgs mechanism and symmetry breaking (Riemannian geometry of discrete spaces)... Nowadays, physicists use the methods of non-commutative geometry in physical systems (gap labelling [6], quantum-Hall effect [6], quantum field theory [7], etc. ).

It should be clear that every physicist working in quantum physics works in noncommutative geometry (but often like Monsieur Jourdain). Many mathematical tools developed (or "mathematical facts" discovered) by theoretical physicists do exist and wait to be incorporated in a general and appropriate mathematical framework. Conversely, many tools recently invented by mathematicians wait for being used in quantum physics. It may be sad to remember that, except in dimension two, the whole apparatus of quantum field theory is most of the time a formal series construction whose fundamental mathematical 
significance is rather unclear - maybe non-commutative geometry will cast a brilliant light on this subject. Again, this is hope.

\section{Non-commutative measure and topology}

We already mentioned the fundamental Gelfand correspondence between topological spaces (resp. compact topological spaces) and commutative $C^{*}$ algebras (resp. $C^{*}$ unital algebras). By removing the word "commutative", we see that non-commutative topology is nothing else than the study of non-commutative $C^{*}$ algebras.

In the classical case -i.e. in the case of "commutative geometry" - and inasmuch as measure theory is concerned, we are usually happy with Borelian measures, i.e. rather than developing abstract sigma algebras and their like from scratch, one first defines a topological structure on a space $X$ and takes as measurable sets only those that are obtained from the topology of $X$. In other words, one considers the sigma algebra generated by the family of open sets. Then, rather than starting from the space $X$, we can start from the algebra of continuous functions (that encodes the topology) and define (positive) measures as (positive) continuous linear forms on the commutative algebra $C(X)$ of continuous functions - i.e. linear functionals $\mu$ such that $\mu(\bar{f} f) \geq 0 \forall f \in C(X)$. The correspondence with the usual definition of measures is obtained via the Riesz theorem, namely by writing $\mu[f]=\int_{X} f d \mu$. From $C(X)$, we build the measures $\mu$. From a given $\mu$, we can build the Hilbert space $\mathcal{H}=L^{2}(X, \mu)$ of square integrable functions. From $\mathcal{H}$, one builds the commutative von Neumann algebra $L^{\infty}(X, \mu)$ of essentially bounded, measurable functions, and this space can be seen as a subspace of $\mathcal{L}(\mathcal{H})$, the algebra of all bounded operators in $\mathcal{H}$. This is done as follows: the elements of $C(X)$ act on $\mathcal{H}$ by multiplication, therefore we obtain a representation $\pi$ in $\mathcal{H}$ of this commutative algebra. The algebra $L^{\infty}(X, \mu)$ is defined as the commutant of $\pi$, i.e. as the set $\{T \in \mathcal{L}(\mathcal{H})$ s.t. $T \pi(f)=\pi(f) T, \forall f \in C(X)\}$. Notice that this algebra is equal to its own commutant in $\mathcal{L}(\mathcal{H})$, so that $C(X) \subset \mathcal{M} \doteq \mathcal{L}^{\infty}(X, \mu)$. The definition of the measure $\mu$ is then extended to $\mathcal{L}^{\infty}(X, \mu)$. All this is standard measure theory, but the point is that it is possible - as we saw - to present it in a way that starts from the algebra and not from the space.

By mimicking the previous construction and removing the qualifier "commutative" we discover "non-commutative measure theory", which therefore coincides with the study of von Neumann algebras. One sketches this construction as follows. We first start from a $C^{*}$ algebra $A$, in general non-commutative - hence it is not the algebra of complex functions on a topological space!- and define states on $A$ - the analogue of measures - as normalized positive linear forms on $A$, i.e. $\mu \in A^{*}, \mu\left(f^{*} f\right) \geq 0, \forall f \in A, \mu(1)=1$. We then construct a Hilbert space $\mathcal{H}$ by first defining a scalar product $\langle f, g\rangle=\mu\left(f^{*} g\right)$ making $A$ a pre-Hilbert space and calling $\mathcal{H}$ the corresponding completed, separated vector space. This is the socalled GNS (Gelfand-Naimark-Segal) construction. As in the commutative case, $A$ acts on $\mathcal{H}$ by multiplication, which defines a representation of $A$ in the space $\mathcal{L}(\mathcal{H})$ of all bounded operators. One finally builds the von Neumann algebra $\mathcal{M}$ as the bi-commutant of $\pi(A)$. Here it is necessary to take the bi-commutant because in the non-commutative case, the commutant and bi-commutant of $\pi(A)$ differ. This $\mathcal{M}$ is therefore the non-commutative analogue of $\mathcal{L}^{\infty}(X, \mu)$. The final step is to extend the definition of $\mu$ to the whole of $\mathcal{M}-$ of course $A \subset \mathcal{M}$, and remember that, by definition, a von Neumann algebra is a unital subalgebra of $\mathcal{L}(\mathcal{H})$ that is equal to its bi-commutant and is endowed with a star operation. 
Remember also that if $A$ is an algebra of operators, $A, A^{\prime}$ and $A^{\prime \prime}$ are usually different but $A^{\prime \prime \prime}=A^{\prime}$.

One should now discuss many other interesting topics, but we shall only mention a few. For example, the non-commutative analogue of infinite positive measures is the theory of weights on von Neumann algebras. This theory incorporates several nice mathematical constructions such as the theory of KMS states, the theory of Tomita-Takesaki, the classification of factors, etc. , and is at the root of statistical mechanics of infinite dimensional systems. Another example is the non-commutative analogue of the theory of embeddings, immersions or isotopy of manifolds: rather than "inserting" spaces (or commutative algebras) within one another, one studies the relative "positions" of several non-commutative algebras. Here we find therefore notions like entropy, completely positive maps, subfactors etc. Physics-wise, this kind of topic is very much related to information loss when we choose one or another-one algebra to describe the same "reality" therefore it is also related to the correspondence between quantum versus classical description [8]. At the fringe between mathematics and physics, let us mention also the new developments in knot theory [9], motivated by questions about relative positions of subfactors.

\section{Non-commutative universal differential forms}

2.1 First definition of $\Omega A$

Given an arbitrary algebra $A$, one can construct a universal object $\Omega A$ as follows. To every element $a \in A$ we associate a new symbol $\delta a$. As a vector space, $\Omega A$ is the linear span of words built out of the symbols $a$ and $\delta a$. Multiplication of two such words is done by concatenation and one imposes the usual algebraic rules of associativity and distributivity over + . The only non trivial relation is the Leibniz rule

$$
\delta(a b)=\delta(a) b+a \delta(b)
$$

Thanks to this rule it is clear that any element can be written as a sum of monomials of the kind $a_{0} \delta a_{1} \delta a_{2} \ldots \delta a_{n}$ or $\delta a_{1} \delta a_{2} \ldots \delta a_{n}$. For instance

$$
\begin{aligned}
a_{0} \delta\left(a_{1}\right) \delta\left(a_{2}\right) a_{3} & =a_{0} \delta\left(a_{1}\right) \delta\left(a_{2} a_{3}\right)-a_{0} \delta\left(a_{1}\right) a_{2} \delta\left(a_{3}\right) \\
& =a_{0} \delta\left(a_{1}\right) \delta\left(a_{2} a_{3}\right)-a_{0} \delta\left(a_{1} a_{2}\right) \delta\left(a_{3}\right)+a_{0} a_{1} \delta\left(a_{2}\right) \delta\left(a_{3}\right)
\end{aligned}
$$

It is convenient to add a unit 1 to $A$, even if it had already a unit - that we call $e$ - and set $\delta 1=0$; then the two kinds of basic "words" defined above collapse because we can write $\delta a_{1} \ldots \delta a_{n}=1 \delta a_{1} \delta a_{2} \ldots \delta a_{n}$. In the extended algebra $e$ is no longer a unit but a projector. The symbol $\delta$ is now defined as an operator by the rules $\delta\left(a_{0} \delta a_{1} \ldots \delta a_{n}\right) \doteq \delta a_{0} \delta a_{1} \ldots \delta a_{n}$ and $\delta^{2}=0$. The $\Omega A$ is graded, since we can write $\Omega A=\sum_{p=0}^{\infty} \Omega^{p} A$, where $\Omega^{p}$ is the linear span of monomials $a_{0} \delta a_{1} \ldots \delta a_{p}$. It is therefore a graded differential algebra. It is a universal object in the sense that it factorizes derivations (see for instance [3]). We should mention that there exists a $Z_{2}$-graded version of $\Omega A$ [10] but we shall not use it. Let us mention also (we shall use this fact) that in many interesting cases $\Omega A$ can be represented - in some cases as an algebra, in other cases as a differential algebra - within the space $\mathcal{L}(\mathcal{H})$, of bounded operators on some Hilbert space $\mathcal{H}$. 


\subsection{Classical example $A=C(X)$}

In that case, $f \in A$ is a function of one variable and we may consider $\delta f$ as a function of two variables, setting $\delta f(x, y)=f(y)-f(x)$. The Leibniz rule is satisfied, since

$$
f(y) g(y)-f(x) g(x)=[f(y)-f(x)] g(y)+f(x)[g(y)-g(x)]
$$

therefore $\delta(f g)=\delta(f) g+f \delta(g)$. In the same way, a monomial like $a_{0} \delta a_{1} \delta a_{2}$ can be interpreted as $a_{0}(x)\left[a_{1}(y)-a_{1}(x)\right]\left[a_{2}(z)-a_{2}(y)\right.$, i.e. as a function of $X \times X \times X$ vanishing on contiguous diagonals. There is a useful map sometimes called the "classical map". It goes from the universal differential algebra $\Omega A$ to the usual differential algebra $\Lambda(X)$ of differential forms on $X$, and is defined on monomials (for instance of degree two) by $C l\left(a_{0} \delta a_{1} \delta a_{2}\right)=a_{0} d a_{1} \wedge d a_{2}$. Notice that $C l(f(x, y))=\left(\partial f(x, y) / \partial y^{\mu}\right)_{y=x} d x^{\mu}$.

\subsection{Second definition of $\Omega A$}

The previous example, and in particular the equation $\delta f(x, y)=f(y)-f(x)$, suggests the following alternative construction of $\Omega A$ for an arbitrary algebra $A$ with unit. For $b \in A$ we set $\delta b=1 \otimes b-b \otimes 1 \in A \otimes A$. Then one gets $a \delta b=a \otimes b-a b \otimes 1 \in \operatorname{ker}(m) \subset A \otimes A$. Here $\operatorname{Ker}(m)$ denotes the kernel of the multiplication map $m(x, y)=x y \in A$. One then defines $\Omega^{1}=\operatorname{Ker}(m)$. As an example of multiplication we choose to consider for instance

$$
\begin{aligned}
a \delta b \delta c & =(a \otimes b-a b \otimes 1)(1 \otimes c-c \otimes 1) \\
& =a \otimes b \otimes c-a b \otimes 1 \otimes c-a \otimes b c \otimes 1+a b \otimes c \otimes 1 .
\end{aligned}
$$

One therefore defines $\Omega^{p}=\Omega^{1} \otimes_{A} \Omega^{1} \otimes_{A} \ldots \otimes_{A} \Omega^{1}$.

\subsection{Usefulness of $\Omega A$}

First we have the universality property - this was already mentioned. But moreover it is possible to consider multilinear forms on $A$ as linear forms on $\Omega A$, which is often very handy. Indeed the knowledge of the $(n+1)$-plet of elements $\left\{a_{0}, a_{1}, \ldots, a_{n}\right\}$ of $A$, where none of the $a_{i}$ is equal to the "extra unit" 1 (remember that we added a unit in paragraph 2.1 ), is totally equivalent to the knowledge of the element $a_{0} \delta a_{1} \ldots \delta a_{n}$ of $\Omega A$. Indeed the symbol $\delta a$ is merely another copy of the symbol $a$. However this is true only before we add the unit 1 since $\delta 1=0$ and, consequently, $\delta a$ may be equal to $\delta b$ without $a$ being equal to $b$. Therefore if $\phi$ denotes a multi-linear form on $A$ - where $A$ denotes the algebra of interest before the addition of an extra unit 1 -, it can be identified with a linear form also called $\phi$ on the universal differential algebra through the relation

$$
\phi\left(a_{0}, a_{1}, \ldots, a_{n}\right)=\phi\left(a_{0} \delta a_{1} \ldots \delta a_{n}\right)
$$

but this form is such that $\phi\left(1 \delta a_{1} \ldots \delta a_{n}\right)=0$. Therefore many concepts - in particular cohomological concepts - related to multilinear forms on $A$ (an algebra with or without unit $e$ ) can be expressed in terms of those linear forms on $\Omega A$ (where we have added an extra unit 1) that satisfy the above constraint. Such linear forms are called "closed" in [3] but we shall avoid this terminology because of the two big amount of possible (co)homological concepts related to the subject. We conclude this subsection by mentioning that $(\mathrm{n}+1)$-linear forms on $A$ or, equivalently, linear forms on $\Omega A$ that vanish on elements $1 \delta a_{1} \ldots \delta a_{n}$ are usually called Hochschild co-chains (more about it later). 


\subsection{The Cuntz and Zekri algebras}

Although we have no room for discussing these two important constructions here, we want to remind the reader that if $u$ is an endomorphism of $A$, i.e. if $u(a b)=u(a) u(b)$ then writing $u=1+q$ defines an operator satisfying the relation $q(a b)=(q a) b+a(q b)+(q a)(q b)$, which looks like a deformed differential and can also be written $q(a b)=q(a) b+u(a) q(b)$. This means that $q$ is a derivation twisted by an endomorphism. One can [11] then construct an algebra $Q A$ out of symbols $a_{0} q\left(a_{1}\right) q\left(a_{2}\right) \ldots q\left(a_{n}\right)$ exactly as we $\operatorname{did}$ for $\Omega A$. There is also a $Z_{2}$-graded version of this construction [12] (the algebra $Z A$ ). Both algebras play a major role in non-commutative geometry.

3. Non-commutative De Rham currents: Hochschild cohomology

We already saw that in the classical case, i.e. $A=C(X)$, the algebra $\Omega A$ was much larger than the algebra $\Lambda(X)$ of differential forms. It can be shown that Hochschild homology coincides, in this case with differential forms. However, because we choose to work with cohomology than with homology, we expect that Hochschild cohomology will coincide with the dual of differential forms (distributions) i.e. with what is called the complex of De Rham currents. This is indeed so. The purpose of this section is therefore to sketch a construction that coincides with the definition of (distributions on) differential forms, but that can be generalized to the case of a non-commutative algebra $A$. In the last section, we shall also describe another construction, leading directly to a non-commutative analogue of $\Lambda(X)$.

\subsection{General construction}

We know that $\delta$ on $\Omega A$ is almost trivial from the homological point of view (only "almost" because $\delta 1=0$ ).

One defines the Hochschild co-boundary operator $b$ as follows.

Acting on $\phi\left(a_{0}, a_{1}, \ldots, a_{n}\right)$ it gives

$$
[b \phi]\left(a_{0}, a_{1}, \ldots, a_{n+1}\right)=\sum_{j=0}^{n}(-1)^{j} \phi\left(a_{0}, \ldots, a_{j} a_{j+1}, \ldots, a_{n+1}\right)+(-1)^{n+1} \phi\left(a_{n+1} a_{0}, \ldots, a_{n}\right)
$$

For example

$$
[b \phi]\left(a_{0}, a_{1}, a_{2}, a_{3}\right]=\phi\left(a_{0}, a_{1}, a_{2} a_{3}\right)-\phi\left(a_{0}, a_{1} a_{2}, a_{3}\right)+\phi\left(a_{0} a_{1}, a_{2}, a_{3}\right)-\phi\left(a_{3} a_{0}, a_{1}, a_{2}\right)
$$

At this point it may be enlightening to compare this expression with the calculation of $a_{0} \delta\left(a_{1}\right) \delta\left(a_{2}\right) a_{3}$ done in section 2.1. Indeed one can see that, provided we identify multilinear forms on $A$ and linear forms on $\Omega A$ (with the constraint discussed previously), calculating $b \phi$ amounts, in this example, to calculating $\phi\left(\left[a_{0} \delta a_{1} \delta a_{2}, a_{3}\right]\right)$. This can be generalized, but we shall not use this formalism and refer to [3] or [10].

The next step is to show that $b^{2}=0$, and this is straightforward and cumbersome...

Since we have a cohomological operator, we define, as is usual, the space of Hochschild cocycles $Z^{n}=\left\{\phi \in C^{n} / b \phi=0\right\}$, the space of Hochschild coboundaries $B^{n}=\left\{\phi \in C^{n} / \phi=\right.$ $b \psi$ for $\left.\psi \in \mathrm{C}^{\mathrm{n}-1}\right\}$ and the cohomology group $H^{n}=Z^{n} / B^{n}$. In the above, $C^{n}$ denotes the space of $n+1$ multilinear forms on $A$ : notice the shift by one unit.

Terminological remark: the curious reader looking for a definition of Hochschild cohomology in a book of homological algebra could be puzzled because this cohomology is 
usually defined as a cohomology with value with a bi-module. Here the bi-module is the dual of $A$ itself, and we did not mention this before because it was not necessary. This choice, along with the fact that we have an obvious pairing between $A^{*}$ and $A$ is, however, at the origin of the above construction.

\subsection{The classical case}

We know that De Rham currents are distributional forms, i.e. if $C$ is a $p$-current and $\omega$ is $p$-form, then $\langle C, \omega\rangle$ is a number. We shall indicate the correspondence between (arbitrary) currents and Hochschild cocycles in the particular case of two-forms, leaving to the reader the task of generalizing (see [3]).

From currents to Hochschild cocycles: $C$ being given, we construct $\phi(f, g, h) \doteq$ $\langle C, f d g \wedge d h\rangle$. One can then check that $b \phi=0$

From Hochschild cocycles to currents: $\phi$ being given, we construct $\langle C, f d g \wedge d h\rangle \doteq$ $\phi(f, g, h)-\phi(f, h, g)$.

The above two formulae are different because there is no reason for $\phi$ to be antisymmetric.

It remains to be seen, $\phi$ being a Hochschild coboundary, if the corresponding current just vanishes, which is an easy consequence of the definition of $b$ and of anti-commutativity of the wedge product.

The conclusion is that the Hochschild cohomology group of degree $p$ coincides with the space of De Rham currents of degree $p$. In particular, one can check that the space $H^{p}$ is trivial as soon as $p$ is larger than $\operatorname{dim}(X)$.

We shall see in section 10 a new construction that, when applied to $A=C(X)$, gives directly the algebra $\Lambda(X)$ of differential forms.

4. Non-commutative De Rham cohomology: cyclic cohomology

One motivation for looking for a more refined cohomology in the non-commutative case is the following: We know, thanks to the previous construction, how to define a non-commutative analogue of differential forms, but we have no candidate - yet - for an analogue of the De Rham cohomology - i.e. Betti numbers etc. Since we are working at the dual level, we are looking for a non-commutative analogue of the operator $\partial$ that acts usually on currents as follows: $\langle\partial C, \omega\rangle=\langle C, d \omega\rangle$, where $\omega \in \Lambda(X)$.

The easiest definition (this is obviously a matter of taste) is the following. We start from the following notion

$$
\phi \text { is cyclic } \Leftrightarrow \phi\left(\mathrm{a}_{0}, \mathrm{a}_{1}, \ldots, \mathrm{a}_{\mathrm{n}}\right)=(-1)^{\mathrm{n}} \phi\left(\mathrm{a}_{\mathrm{n}}, \mathrm{a}_{0}, \mathrm{a}_{1}, \ldots, \mathrm{a}_{\mathrm{n}-1}\right) .
$$

We then make the following fundamental remark [3]: If $\phi$ is cyclic, then $b \phi$ is also cyclic.

It is then natural to consider the cyclic subcomplex of the Hochschild complex, i.e. to consider only cyclic Hochschild co-chains along with the same $b$ operator as before. One then defines the spaces $Z_{\lambda}^{n}, B_{\lambda}^{n}$ of cyclic cocycles, cyclic coboundaries and their quotients, the cyclic cohomology groups $H_{\lambda}^{n}$.

In the "classical case", i.e. with $A=C^{\infty}(X)$, one [3] proves

$$
H_{\lambda}^{k}=\operatorname{Ker\partial } \oplus H_{k-2} \oplus H_{k-4} \cdots
$$

where $K e r \partial$ is the kernel of the $\partial$ operator in the space of $k$-dimensional currents and where $H_{p}$ denotes the homology group of degree $p$ for currents. This means that we do 
not get a term-by-term equality between the cyclic cohomology groups and the De Rham homology groups for currents, but that, nevertheless, the information content is the same, since, provided we choose $k$ big enough, the even or odd cyclic cohomology groups are equal to the direct sum of all the (even or odd) De Rham homology groups.

This result suggests, at least in the case of $C(X)$, that there should be a canonical way of sending $H_{\lambda}^{p}$ in $H_{\lambda}^{p+2}$. This is indeed true. Actually, a much stronger property holds: for any algebra one can define an operator $S$, often called the Connes ' periodicity operator, that maps $C_{\lambda}^{p}$ to $C_{\lambda}^{p+2}$ - with $C_{\lambda}^{*}$ referring to cyclic cochains. It is therefore natural to define, in general, the so-called "even and odd periodic cyclic cohomology groups" as the inductive limit of $H_{\lambda}^{2 p}$ and $H_{\lambda}^{2 p+1}$.

\section{A bestiary of other nice concepts}

We have not enough time here to present all the inter-relations between the above constructions and to mention the many other nice concepts and operators introduced in [3]. We shall only give the definition of those operators that play (or bound to play) an important role in non-commutative geometry. Besides the periodicity operator $S: C_{\lambda}^{p} \rightarrow$ $C_{\lambda}^{p+2}$ that was already mentioned, we may consider the following operators:

the cyclic antisymmetrization operator

$$
\begin{aligned}
{[A \phi]\left(a_{0}, a_{1}, \ldots, a_{n}\right) } & =\phi\left(a_{0}, a_{1}, \ldots, a_{n}\right)+(-1)^{n} \phi\left(a_{n}, a_{0}, \ldots, a_{n-1}\right) \\
& +\phi\left(a_{n-1}, a_{n}, a_{0}, \ldots\right)+(-1)^{n} \phi\left(a_{n-2}, a_{n-1}, a_{n}, a_{0}, a_{1}, \ldots\right)+\ldots
\end{aligned}
$$

, and the non-antisymmetrized boundary operator $B_{0}$ defined as

$$
\left[B_{0} \phi\right]\left(a_{0}, a_{1}, \ldots, a_{n}\right)=\phi\left(e, a_{0}, \ldots, a_{n}\right)-\phi\left(a_{0}, \ldots, a_{n}, e\right)
$$

where $e$ is the unit of the algebra which in this case is required to be unital.

The cyclic boundary operator is, by definition, $B \doteq A B_{0}$. Then $B$ maps $C^{n}$ to $C^{n-1}$ and one can show that $B^{2}=0$ and that $b B+B b=0$. Using the last two properties along with $b^{2}=0$ one can also define a bi-complex (notice that $b$ and $B$ act in opposite directions - from $p$ to $p+1$ or the converse - so that $b B+B b$ sends $p$ chains to $p$ chains). From this bi-complex one can define "entire cyclic cohomology" [13] as follows. Entire cocycles are sequences $\left(\phi_{2 n}\right)$ or $\left(\phi_{2 n+1}\right)$ made of even or odd functionals $\phi$ and satisfying the following constraint (we write it below for the odd case):

$$
b \phi_{2 n-1}+B \phi_{2 n+1}=0 .
$$

Using such a cocycle, also assumed to obey some kind of growth conditions, one can define an entire function on the algebra $A$,

$$
F_{\phi}(x)=\sum_{n=0}^{\infty} \frac{(-1)^{n}}{n !} \phi_{2 n}(x, x, \ldots, x) .
$$

There are explicit formulae for such cocycles $-c f$. [7],[13] and the talk by K.Osterwalder (these proceedings). Notice that such an $F_{\phi}$ can be thought of as a kind of "effective 
action". Entire cyclic cohomology seems to provide the appropriate formalism needed to handle infinite-dimensional cases in "commutative geometry".

6. Non-commutative vector bundles and connections

Again we use classical geometry as a guide. A principal bundle is nothing else than a "machine" helping us to construct associated - in particular vectorial - bundles. These vector bundles, in turn, are used to construct sections of vector bundles - i.e. physical matter fields. The important algebraic remark is then the following : The space $\mathcal{E}$ of sections of a vector bundle $E$ above a manifold $X$ is a module over the algebra $A=C^{\infty}(X)$. Finally we know that anything that is twisted can be untwisted by putting it into a space of higher dimension. In bundle language, this means that any vector bundle - trivial or not - can be trivialized by increasing its dimension (adding copies of $C^{n}$ ).

In non-commutative geometry, a "vector bundle" will be replaced by a left or right module over a non-necessarily commutative algebra $A$. The "left" or "right" adjective comes from the fact that, in a general situation, a module over $A$ will be left or right but not necessarily a bi-module. In other words, if $v \in \mathcal{E}$ and $f \in A, f . v$ and $v$. $f$ will not both make sense as elements of $\mathcal{E}$. Actually, and because of our western habits of writing from left to right, it turns out that it is usually much more convenient to consider right modules than left modules. A trivial bundle will now be a free module, i.e. a module isomorphic with $A^{n}$ for some $n$. A non trivial "non-commutative vector bundle" will be characterized by the fact that it is a quotient of a free module, i.e. $\mathcal{E}$ is isomorphic with $p A^{n}$ for some projector $p, p^{2}=p$. This is the non-commutative analogue of the "untwisting property" that was recalled above. To summarize: non-commutative vector bundles are (right) finite projective modules over an algebra $A$. The classification of vector bundles above a space $X$ leading to the so-called $K$ theory of $X$ is now replaced by classification of projectors (modulo appropriate equivalence) and this defines the $K$-theory of the algebra $A$. This is less elementary stuff and we stop the discussion here.

To define connections and covariant exterior derivatives, we choose again the classical situation as a guide. The covariant exterior differential $\nabla$ is a map $\mathcal{E} \rightarrow \mathcal{E} \otimes_{A} \Lambda^{1}$ sending vector fields (or tensor fields) to 1 -form-valued vector (tensor) fields. It satisfies

$$
\nabla(X f)=(\nabla X) f+X \otimes d f, X \in \mathcal{E}, f \in A .
$$

One then extends this definition to $\mathcal{E}^{p}=\mathcal{E} \otimes_{A} \Lambda^{p}$ as follows

$$
\begin{aligned}
\nabla: \mathcal{E}^{p} & \rightarrow \mathcal{E}^{p+1} \\
\nabla(X \otimes \lambda) & =(\nabla X) \lambda+(-1)^{p} X \otimes d \lambda, X \in \mathcal{E}^{p}, \lambda \in \Lambda .
\end{aligned}
$$

One then checks that the curvature $\nabla^{2}$ is a linear object:

$$
\nabla^{2}(X \lambda)=\left(\nabla^{2} X\right) \lambda, \lambda \in \Lambda
$$

In non-commutative geometry, we do therefore exactly the same! The only points to which one should pay attention are the following. First we use right multiplication by functions (as in above formulae) since $\mathcal{E}$ is only a right $A$-module. Next, one has to choose a graded differential algebra $\Lambda$ that should be a $A$-bimodule. In the classical case, $\Lambda$ the 
algebra of differential forms $\Lambda(X)$ - with $A=C^{\infty}(X)=\Lambda^{0}(X)$. In the non-commutative case, there are many possibilities and each one defines a differential calculus. The most obvious choice is to choose $\Lambda=\Omega A$, as defined in section 2 (notice that even in the classical case, this possibility leads to a generalization of the usual differential calculus, since the algebra of differential forms is only a quotient of the universal algebra $\Omega A$ ). When the algebra $A$ has enough derivations, it may be useful to build a differential calculus relying on the choice $\Lambda=C(\operatorname{Der} A, A)$ of $A$-valued multilinear forms on the space of derivations, or even on the choice of $\Omega_{D V} A$, its smallest graded differential subalgebra containing $A$. This possibility was investigated in [14] and [15]. Still another choice will be briefly described in the last section. Notice, finally, that in the case where $A$ is equipped with an extra structure - for example if $A$ is an Hopf algebra - there may be particular differential calculi enjoying particularly nice properties [16].

As a basic example we may take $\mathcal{E}=A$ itself and $\Lambda=(\Omega A, \delta)$. We suppose that $A$ is unital (maybe after adding a unit). Let $1 \in A$ and call $\omega \doteq \nabla 1 \in \Omega^{1}$. Let $f \in A$ then $\nabla f=\nabla(1 f)=(\nabla 1) f+1 \otimes \delta f=\delta f+\omega f$. The curvature is $\theta \doteq \nabla \omega=\nabla^{2} 1$, and therefore $\theta=\nabla(1 \omega)=(\nabla 1) \omega+(-1)^{0} 1 \delta \omega=\delta \omega+\omega^{2}$

Notice that even in the "classical case" $A=C(X)$, we may consider connections such as $\omega \doteq f \delta f-\frac{1}{2} \delta\left(f^{2}\right)$ which are non-zero in $\Omega^{1}$ and therefore are connections in the previous sense, but have no classical counterpart since $C l(\omega) \in \Lambda^{1}$ is obtained by $C l(\omega)=f d f-\frac{1}{2} d f^{2}=0$. Remember that an arbitrary element of $\Omega^{1}$ can be defined as a function of two variables vanishing on the diagonal, hence it is a bi-local quantity.

7. Non-commutative elliptic operators

Motivations. People want to be able to "compute" cyclic cocycles and also to be able to consider "abstract" elliptic operators, generalize index theory to the non-commutative case, etc.

Almost trivial remarks. Consider a $Z_{2}$-graded Hilbert space $\mathcal{H}=\mathcal{H}_{+} \oplus \mathcal{H}_{-}-$think for instance of the left and right spinor fields on a $2 n$-dimensional spin manifold - with grading $\Gamma$ equal to $\left(\begin{array}{cc}1 & 0 \\ 0 & -1\end{array}\right)$ - think of the chirality operator $\gamma_{5}$ - with an algebra $A$ acting on $\mathcal{H}$ - think of the multiplication of spinor fields by scalar functions defined on the manifold. Then, every operator $B$ in $\mathcal{H}$ can be decomposed into an even and odd part $B=B_{+}+B_{-}$ with $B_{ \pm} \doteq \frac{1}{2}\left(B \pm B^{\Gamma}\right), B^{\Gamma} \doteq \Gamma B \Gamma$. Let us now consider an operator $F: \mathcal{H} \rightarrow \mathcal{H}$ which obeys the properties $F^{2}=1$ and $F \Gamma=-\Gamma F$. Then, let us define

$$
d B=i[F, B]_{g} \text { with }[\mathrm{F}, \mathrm{B}]_{\mathrm{g}}=\mathrm{FB}-\mathrm{B}^{\Gamma} \mathrm{F}
$$

It is easy to check that the $d$ operator, defined thanks to this graded commutator, is a derivation and satisfies the property $d^{2}=0$ (coming from $F^{2}=1$ ). As a classical example, we may choose the Dirac operator $D$ on a Riemannian manifold, write a polar decomposition $D=|D| F$ where $|D|$ is positive and $F^{2}=1$.

Non-commutative generalization. The only thing to be done in order to go to the non-commutative framework is to forget about commutativity, spinor fields, chirality and $A=C(X)$ but to promote the previous properties to the status of a definition - technically, one has also to assume that $[F, a]$ is a compact operator for any $a$ in $A$. Such a triple $(\mathcal{H}, \Gamma, A)$ then defines what is called an "even Fredholm module" [3]. There is an analogous theory for the odd case which, in the classical case, corresponds to the choice of a manifold 
with odd dimension -when there is no chirality operator and there are no half-spinors. Let us proceed with the study of the even case. The interest of this construction is the following. One can prove [3] that

$$
\tau\left(a_{0}, a_{1}, \ldots, a_{n}\right)=\operatorname{Str}\left(a_{0}\left[F, a_{1}\right]\left[F, a_{2}\right] \ldots\left[F, a_{n}\right]\right),
$$

where

$$
\operatorname{Str}(B) \doteq \frac{1}{2} \operatorname{tr}(\Gamma F[F, B])
$$

is a cyclic cocycle on $A$. This makes sense provided the trace exists, which implies that $n$ should be large enough, say $n>p-1$. In that case, the even Fredholm module is called "p-summable". The previous construction therefore yields cyclic cocycles i.e. what we may call topological invariants of the algebra $A$. From that point one can develop a non-commutative analogue of index theory, of the K-theory and of its dual, called Khomology. Going into these matters would drive us too far, but just note that, exactly as in the classical case where one gets "topological numbers" from the pairing between vector bundles and operators, one gets also topological numbers in the non-commutative case - like for instance $\tau(e, \ldots, e)$ - from the pairing between an abstract operator $F$ used to define $\tau$ - and a "non-commutative vector bundle" characterized by the choice of a projector $e$ in $A$ or in $M_{n}(C) \otimes A$.

\section{Non-commutative Riemannian Geometry: K-cycles}

The classical path. The usual approach in textbooks of differential geometry is to follow the path : topological space, smooth manifold, metric (and distance), Dirac operator, spinor fields. Not surprisingly, if we try to express everything by using functions on the manifold $X$ - that we shall assume compact here - rather than in terms of points, we may follow exactly the same steps but in the reverse order. More precisely, the claim [2] is that we can recover everything from the data $(\mathcal{A}, \mathcal{H}, D)$ where $\mathcal{A}$ is an Abelian von Neumann algebra of multiplication by measurable bounded functions on $X$, where $\mathcal{H}$ is the Hilbert space of $L^{2}$ spinors and where $D$ is the Dirac operator. How can we proceed (without knowing $X$, of course!)? First we call $\mathbf{A}$ the subset of $\mathcal{A}$ containing all $f \in \mathcal{A}$ such that the commutator $[D, f]$ is bounded. It can be shown that such functions $f$ are almost everywhere equal to a Lipschitz function - i.e. $|f(x)-f(y)| \leq c d(x, y), \forall x, y \in X$. This remark can be used to define the distance itself as

$$
d(x, y)=\operatorname{Sup}\{|f(x)-f(y)|, f \in \mathcal{A},|[D, f]| \leq 1\} .
$$

Notice that a supremum over a space of functions replaces the usual infimum over a space of points. The final step is to recover the $C^{*}$ algebra $A$ of continuous functions on $X$ as the norm closure of $\mathbf{A}$ in $\mathcal{A}$. Now we have $A$ and can therefore reconstruct the space $X$ itself as it was recalled in the first section.

In the non-commutative case, the whole construction goes through, just by removing the adjective "commutative". More precisely, one start from the same data $(\mathcal{A}, \mathcal{H}, D)$ where the von Neumann algebra $\mathcal{A}$ is not supposed to be Abelian - these data are called a "K-cycle" in [2]. Then $\mathbf{A}$ and the- non-commutative $-C^{*}$ algebra $A$ are constructed as above. The distance $d$ is also obtained from the same formula, but now the "points" 
$x$ and $y$ are states on the algebra. We have therefore the non-commutative analogue of a Riemannian structure (and we write $x[f]$ rather than $f(x)$ since we have states, i.e. generalized "points".)

The last missing concept is the non-commutative analogue of the volume form associated with a Riemannian metric. The crucial remark - valid in the classical case - is that we may write

$$
\int f d v=\operatorname{Tr}_{\text {Dixmier }}\left(f D^{-n}\right)
$$

where $f \in A, n=\operatorname{dim}(X)$ and where the Dixmier trace is defined - formally - as a limit of the sequence $\frac{1}{\log N} \Sigma_{0}^{N} \lambda_{j}, \lambda_{j}$ running over the eigenvalues of the operator $f D^{-d}$. The whole point is that this "trace", which is a trace but gives zero on any operator of trace class, is a concept that also makes perfect sense when $f$ belongs to a non-commutative algebra $A$. In physicists' language, this "Dixmier trace" picks up the pole part of a logarithmically divergent operator. Notice that $n$ does not need to be an integer. The above formula is then used to define the left-hand side, i.e. the non-commutative analogue of the volume form. Notice that this formula is rather intuitive - at least in a very formal sense -for instance by writing $d v=d^{4} x$ and $D=\gamma^{\mu} \partial / \partial x^{\mu}$ ! To conclude we notice that the whole geometry - including the volume form - has been recovered from the single piece of data called a"K-cycle".

9. The non-commutative Yang-Mills action

Let $D$ be a Dirac operator - in the previous sense - and write $D=|D| F$ with $F^{2}=1$. From a formal expression $a_{0} \delta a_{1} \delta a_{2} \ldots \delta a_{n}$, i.e. an element of $\Omega A$, we already know that we can build $\operatorname{Str}\left(a_{0}\left[F, a_{1}\right] \ldots\left[F, a_{n}\right]\right)$ which is a topological invariant. With the previous formalism at our disposal, it is rather natural to consider also a quantity such as $\operatorname{Tr}_{\text {Dixmier }}\left(a_{0}\left[D, a_{1}\right]\left[D, a_{2}\right] \ldots\left[D, a_{n}\right] D^{-n}\right)$. Indeed in a classical and "flat" case, the operator $[D, f]$, with $f \in C^{\infty}(X)$, is nothing else than a multiplication by $\gamma^{\mu} \partial_{\mu} f$, where $\gamma^{\mu}$ are Dirac (Clifford) generators and $n=\operatorname{dim}(X)$. Choosing $\mathcal{E}$ as $A$ itself and the differential algebra $\Omega A$, we see that $\theta$, the curvature of the connection, is an element of $\Omega^{2}$, and $\theta^{2}$ is an element of $\Omega^{4}$, therefore a sum of elements of the kind $a_{0} \delta a_{1} \ldots \delta a_{4}$. One can now represent $A$ and $\Omega A$ as operators in $\mathcal{H}$, thanks to the replacement $\delta a \rightarrow[F, a]$ or $\delta a \rightarrow[D, a]$. Notice that only the first leads to representation of $\Omega A$ as a differential algebra since $D^{2} \neq 1$. In any case, one expects the first kind of expression - using $F$ and $S t r$ - to lead to a topological invariant like $\theta_{\mu \nu} \tilde{\theta}^{\mu \nu}$ and the second kind $-\operatorname{using} D$ and Dixmier trace - to lead to the action functional $\theta_{\mu \nu} \theta^{\mu \nu}$. This is indeed so.

In general, one has to couple the connection $\nabla$ to the Dirac operator $D$, i.e. to consider the analogue of $\gamma^{\mu}\left(\partial_{\mu}-\omega_{\mu}-A_{\mu}\right), \omega_{\mu}$ being the spin connection. We also need a spinor scalar product to be able to write the fermionic action, i.e. we need the analogue of the Dirac "bar" operator. The method consists in chosing an Hermitian (and finite projective) module $\mathcal{E}$ over $A$, along with a connection $\nabla$ as a map from $\mathcal{E}$ to $\mathcal{E} \otimes \Omega^{1}$. This connection has to be compatible with the hermitian structure, in the sense that $d(\langle\psi, \phi\rangle)=\langle\psi, \nabla \phi\rangle-\langle\nabla \psi, \phi\rangle$ - in physics, we prefer to write $\langle\psi, \phi\rangle=\langle\bar{\psi} \phi\rangle$. One then defines $D_{\nabla}$ as a map from $\mathcal{E} \otimes_{A} \mathcal{H}$ to itself such that $D_{\nabla}(v \otimes \psi)=v \otimes D \psi+(\nabla v) \psi$ where $\nabla v$, in this last expression, has to be understood as the linear operator in $\mathcal{H}$ representing the element $\nabla v$ of $\mathcal{E} \otimes \Omega^{1}$. 


\section{Symmetry breaking and Higgs mechanism in non-commutative geometry}

Using non-commutative geometry to recover the usual pure Yang-Mills theory is certainly satisfactory but does not bring much from the conceptual point of view. Situation is different in the case of symmetry breaking (Yang-Mills fields coupled to Higgs fields). The mere replacement of the "classical" algebra $A=C(X)$ by the algebra $A=C(X) \oplus C(X)$ in the previous construction, i.e. the replacement of the space $X$ by a "pair" of such spaces, leads directly to a Yang-Mills action, incorporating not only the usual pure Yang-Mills term but also a kinetic term for Higgs fields and a self coupling via a quartic potential exhibiting symmetry breaking. This was shown in [4]. The definition of the generalized Yang-Mills action was given in the last paragraph. The calculation, using the above algebra $A$, the module $\mathcal{E}=A$ itself, and the differential algebra $\Omega A$, is straightforward. Formally, the obtained expression is a Lagrangian density describing the interaction between a gauge field and a Higgs scalar field, along with a self-coupling of this scalar field via a symmetry-breaking potential of fourth degree. Conceptually, non-commutative geometry unifies together the concepts of Yang-Mills fields and Higgs fields in a generalized connection. In the same way, the "non-commutative" Dirac operator incorporates the usual Dirac operator (describing the coupling of spinors to the metric and to the connection), but also the Yukawa operator (describing the coupling of spinors to Higgs fields and in particular the mass matrix itself). In this sense, the masses and coupling of elementary fermions are encoded in the Riemannian structure of the algebra describing what we call space-time.

Recovering the Standard Model of electroweak interactions in this framework amounts, in particular, to selecting the "right" algebra $A$. The result of the previous calculation suggests that we have to take a direct sum of two algebras $A=A_{1} \oplus A_{2}$, in order to get Higgs fields. When $A_{1}$ and $A_{2}$ are commutative, i.e. $A_{1}=C\left(X_{1}\right)$ and $A_{2}=C\left(X_{2}\right)$, the connection $\omega$, considered as an element of $\Omega^{1}$, is a function of two variables and can be decomposed in three pieces: $\omega\left(x_{1}, y_{1}\right), \omega\left(x_{2}, y_{2}\right), \omega\left(x_{1}, y_{2}\right)$ with $x_{1}, y_{1} \in A_{1}$ and $x_{2}, y_{2} \in A_{2}$. The first two pieces, in the limit where $\left(y_{i}-x_{i}\right)$ goes to zero, become usual connection one-forms. The last cannot be made infinitesimal and becomes the Higgs field (a scalar). Intuitively, one can think of $X_{1}$ and $X_{2}$ as "parallel universes" where left - or right - movers live, or as the two sides of a piece of paper separated by a (here constant) distance $1 / \mu$, the Higgs mass.

Physically, we have to identify the algebras $A_{1}$ and $A_{2}$. Rather than choosing a nontrivial projective module over the algebra $A$, it is computationally simpler to choose a bigger $A$ but take the module as $A$ itself. This is what is proposed in the last version [2] with the choice $A \doteq(H \oplus C) \otimes C(X)$ where $H$ and $C$ are the fields of quaternions and complex numbers. Emergence of the gauge group $S U(2) \times U(1)$ is then very natural. The above algebra $A$ describes a "space" that is almost classical, in the sense that it is the product of a usual manifold times a discrete space (notice that the space of complex functions on the set with two elements is simply $C \oplus C)$. Geometry of the first is specified by a usual Dirac operator, geometry of the second is specified by the Yukawa operator of physicists and encodes in particular the mass matrix (which has no particular reason to be diagonal). Incorporation of quarks and colour degrees of freedom is also described in [2], which means that the classical Lagrangian describing the usual standard model of particle physics is recovered as a particular case of a very general mathematical construction 
which, by far, encompasses the usual classical setting of gauge theories, using principal and vector bundles. Let us notice an important technical detail: in [2], the differential algebra used to develop a differential calculus - as described above - and in particular to recover the Standard Model of electroweak interactions, is not the differential algebra $\Omega A$, but a differential algebra $\Omega_{D} A$ defined as the quotient of the first by a differential ideal, namely by $J \doteq J_{0}+d J_{0}$, where $J_{0} \doteq \operatorname{Ker}(\pi)$ and $\pi$ is the map from $\Omega A$ to $\mathcal{L}(\mathcal{H})$, sending $a_{0} \delta a_{1} \ldots \delta a_{n}$ to $a_{0}\left[D, a_{1}\right] \ldots\left[D, a_{n}\right]$. Actually, $\Omega_{D} A$ is a fundamental object since, in the classical case, it can be shown to be isomorphic with the algebra of differential forms themselves - here one gets directly the forms and not the De Rham currents, unlike in section 3. This slight modification of the construction presented in [4] has several advantages, the main one being of getting rid of spurious (unwanted) terms that were appearing in the calculation of the Higgs potential.

Inspired from [4], another non-commutative construction of the Lagrangian of the Standard Model was proposed in [17]. This construction uses rather simple mathematical tools and is not supposed to be a multi-purpose machine, able to handle any kind of algebraic situation. It was however devised to recover the Standard Model from noncommutative geometry - in particular from the notion of generalized connections - and has the merit of being very simple to grasp and maybe to serve as an introduction to the ideas of [2] summarized here. Our approach can be shown to be essentially equivalent to the last construction presented in [2] (it is not fully equivalent to the one presented in [1] or [4], because of the different choice that was made for the differential algebra, namely $\Omega A$ in [1] (or [4]) and $\Omega_{D} A$ in [2]). Another paper [19], which is more "phenomenological", relates these ideas with older approaches dealing with the appearance of Lie super-algebras in the Standard Model (see also [19]).

The final outcome of [2] (or [18]) is the classical Lagrangian that everybody uses in particle physics. There is an argument about - whether or not - this kind of approach decreases the number of arbitrary constants in the Standard Model. At the classical level, this seems to be the case (or at least there are "canonical" choices leading to such a decrease). But in any case, getting a classical Lagrangian should not be considered as the end of a story but only as its beginning! One has indeed to consider the fully interacting quantum field theory which, unfortunately, exists only in a formal series sense: the principle of gauge invariance alone - the only principle on which renormalization of perturbative gauge theory relies - allows for a number of arbitrary constants that is fixed in the Standard Model. Decreasing this number, i.e. imposing, to all orders of quantum field theory, constraints unrelated with gauge principles, looks rather artificial and would be justified if we had a geometrical - or algebraical - understanding of what the fully interactive (non perturbative) quantum field theory is. This is unfortunately not yet the case and needs an infinite dimensional jump! It may be that such an understanding is not too far ahead and will require part (if not all) of the mathematical concepts reviewed in these notes. 


\section{References}

[1] A. Connes, Introduction à la géométrie non commutative (InterEditions, Paris, 1990).

[2] A. Connes, An introduction to non-commutative geometry (to appear).

[3] A. Connes, Noncommutative differential geometry, Publ. Math. IHES, 62 (1985).

[4] A. Connes, J. Lott. Particle models and non-commutative geometry, Nucl. Phys. B (Proc. Suppl.) 18B,29-47 (1990).

[5] D. Kastler, A detailed account of A. Connes's version of the Standard Model in noncommutative geometry. Marseille CPT preprint (1991), to appear in Rev. Math. Phys.

[6] J. Bellissard, $\mathrm{K}$ theory of $C^{*}$ algebras in solid state physics, statistical physics and field theory, mathematical aspects, Springer Lecture Notes in Physics 257 (1986).

[7] A. Jaffe, A. Lesniewski and K. Osterwalder, Quantum K theory: the Chern character, Commun. Math. Phys. 118 No1,1-14 and 99-156 (1988).

[8] Ph. Blanchard, A. Jadczyk, On interaction between classical and quantum systems, BiBos Preprint (1992).

[9] V. Jones, A polynomial invariant for knots via von Neumann algebras, Bull. Amer. Math. Soc. 12, 103-112 (1985).

[10] D. Kastler, Cyclic cohomology within the differential envelope, Coll. Travaux en Cours (Hermann, Paris 1987).

[11] J. Cuntz, Another view to KK-theory, J. of K-theory, vol. 1, No. 1 (1987).

[12] R. Zekri, A new description of Kasparov's theory of $C^{*}$ algebras extensions, J. Func. Anal. (1988).

[13] A. Connes, Entire cyclic cohomology of Banach algebras and character of $\Theta$-summable modules, J. of K-theory, Vol. 1, No. 6 (1988).

[14] M. Dubois-Violette, Dérivations et calcul différentiel non commutatif, C.R. Acad. Sci. Paris, t. 307, Série I, p. 403-408 (1988).

[15] M. Dubois-Violette, K. Kerner, J. Madore, Classical bosons in a non-commutative geometry, Class. Quant. Grav. 6, 1709 (1989).

[16] S.L. Woronowicz, Differential calculus on compact matrix pseudogroups (Quantum groups), Commun. Math. Phys. 122, 125-170 (1989).

[17] R. Coquereaux, G. Esposito-Farèse, G. Vaillant, Higgs fields as Yang-Mills fields and discrete symmetries, Nucl. Phys. B 353, 689-706 (1991).

[18] R. Coquereaux, G. Esposito-Farese and F. Scheck, The theory of electroweak interactions and $S U(2 \mid 1)$ algebraic superconnections, IHES/P/100, CPT/PE/2464, to appear in Int. J. Mod. Phys.

[19] R. Coquereaux, Yang-Mills fields and symmetry breaking: from Lie super-algebras to non-commutative geometry, talk given at the M. Born German-Polish Symposium, Wojnowice, 1991. CPT-91/PE-2367 